\title{
Placebo Brain Stimulation Affects Subjective but Not Neurocognitive Measures of Error Processing
}

\author{
Michiel van Elk ${ }^{1,2} \cdot$ Eva Groenendijk ${ }^{1} \cdot$ Suzanne Hoogeveen ${ }^{1,2}$ \\ Received: 15 January 2020 / Accepted: 22 April 2020 / Published online: 7 May 2020 \\ (C) The Author(s) 2020
}

\begin{abstract}
The aim of this preregistered EEG study was to show how expectations about enhanced or impaired performance through transcranial stimulation affect feelings of agency and error processing. Using a single-blind experimental design, participants $(N=57)$ were attached to a transcranial direct current stimulation (tDCS) device, and in different blocks, they were verbally instructed to expect enhanced or impaired cognitive performance, or no effects of the brain stimulation. In all cases, but unbeknownst to the participants, we used an inert sham tDCS protocol. Subsequently, we measured their response to errors on a cognitive control task. Our expectancy manipulation was successful: participants reported improved subjective performance in the enhancement compared with the impairment condition - even though objective performance was kept at a constant level across conditions. Participants reported the highest feelings of agency over their task performance in the control condition, and lowest feelings of agency in the impairment condition. The expectancy manipulation did not affect the error-related negativity $(E R N)$ in association with incorrect responses. During the induction phase, expecting impaired versus enhanced performance increased frontal theta power, potentially reflecting a process of increased cognitive control allocation. Our findings show that verbally induced manipulations can affect subjective performance on a cognitive control task, but that stronger manipulations (e.g., through conditioning) are necessary to induce top-down effects on neural error processing.
\end{abstract}

Keywords Neuroenchantment $\cdot$ Brain stimulation $\cdot$ Error-related negativity $\cdot$ Feelings of agency

\section{Introduction}

The emergence of new technologies, such as self-driving cars or bioengineering, often raises issues regarding personal responsibility. Take for instance the example of a patient suffering from obsessive-compulsive disorder (OCD), who has been treated with the implantation of deep brain electrodes (Figee et al. 2013). The brain stimulation may relieve the patient from OCD-related symptoms, but now suppose that the patient would suddenly be found stealing money from a cash register. Could he blame the deep brain stimulation for his morally inappropriate behavior? Or take the example of a student who is using a commercially available brain

Michiel van Elk

m.vanelk@uva.nl

1 Department of Psychology, University of Amsterdam, Nieuwe Achtergracht 129B, 1018 XA Amsterdam, The Netherlands

2 Amsterdam Brain and Cognition Center, University of Amsterdam, Amsterdam, The Netherlands stimulation device for cognitive enhancement. He can attribute improved concentration while studying to the transcranial stimulation, but what would happen when he encounters a negative outcome such as insomnia or restlessness? Would he blame the device for the adverse experiences that he encounters?

To understand the processes underlying these phenomena, we must carefully distinguish between the promises and the pitfalls of "neuroenchantment," which we define as the phenomenon that people are mesmerized by brain-based explanatioAli et al. 2014ns and that they have a strong trust and belief in neuroscientific findings and techniques (; Maij and van Elk 2018). Scholars, reporters, consumers, and the general public alike appear enchanted by the human brain. New techniques like neurostimulation and neurofeedback offer great promises to unleash the hidden powers of the mind and to improve performance (Ali et al. 2014). In the media, there is a lot of attention for cognitive enhancement, e.g., through brain training or through the use of neurostimulation techniques (O'Connor et al. 2012). Building on the widespread belief that the brain is an organ that needs to be 
optimized, companies now even offer commercially available tools and devices for brain improvement (Steenbergen et al. 2016).

In line with people's trust and belief in the efficacy of neurocognitive techniques, in recent studies, we found that manipulating belief in the possibility to unleash one's hidden mental energies through placebo brain stimulation could induce extraordinary experiences, improved subjective performance, and affected neural error processing (Hoogeveen et al. 2018; Maij and van Elk 2018; Maij et al. 2019). Typically, placebo effects refer to the subjective and objective positive effects of an otherwise inert treatment (i.e., pharmacologically, biologically, or technologically inert) that can be attributed to verbal suggestion, conditioning, or the therapeutic context (Benedetti et al. 2011). Nocebo effects in turn refer to the negative consequences experienced through an inert treatment, which can be attributed to negative expectations (e.g., induced through reading about side effects; Colloca and Finniss 2012). Our previous findings using placebo brain stimulation thus indicate that inducing belief in the potential of neurostimulation techniques could maximize placebo responses (i.e., over and beyond classical placebo manipulations such as pills or creams), because (1) belief in brain stimulation optimally exploits people's prior beliefs and motivation, (2) it immerses participants in a seemingly cutting-edge technological environment, and (3) it allows inducing specific expectations regarding the acute and direct effects of brain stimulation. Indeed, meta-analytic studies have shown that placebo responses are stronger for manipulations involving more complex procedures and technologies (Meissner et al. 2013). As such, it has been suggested that sham neurostimulation technologies may be used as a "superplacebo" (Thibault et al. 2017).

On the other hand, belief in the potential for brain stimulation may have adverse consequences, in terms of deferring moral responsibility (Racine et al. 2008; Slaby and Choudhury 2011) — as illustrated by the examples above. The popular use of brain-based explanations of human behavior raises concerns about the autonomy of human behavior (e.g., "my brain made me do it"; cf., Farah 2002; Roskies 2006) and neurostimulation techniques could reduce people's sense of individual agency. In a recent study (Hoogeveen et al. 2018), we found that when participants were expecting impaired performance due to transcranial stimulation, they indeed experienced less agency over errors they committed on a cognitive control task. At a neural level when participants were expecting enhanced performance, they showed a stronger error-related negativity (ERN), a neural marker that has been associated with error monitoring and cognitive control (Falkenstein et al. 1991; Gehring et al. 2018; Gehring et al. 1993), suggesting surprise in response to unfulfilled expectations. In contrast, when they were expecting impaired performance, a stronger externalization of agency was associated with a reduced ERN amplitude - suggesting that errors were taken less into account when participants believed they were externally caused.

The current preregistered study provides a direct highpowered replication and extension of our previous study (Hoogeveen et al. 2018), to assess what happens to people's attribution of agency and their neural response to errors, when they expect enhanced or impaired cognitive performance through transcranial stimulation. In addition to our previous study, here we were specifically interested in the question whether placebo effects using sham brain stimulation are enhanced for highly suggestible participants. We also set out to investigate the neural mechanisms driving the experience of enhanced versus impaired performance. To this end, we equipped healthy adults with a real but inactive (i.e., placebo) brain stimulation device (i.e., a transcranial direct current stimulation (tDCS) electrodes). Participants were verbally instructed that the device was capable of boosting or reducing their brain capacity, thereby affecting their performance on a cognitive control task. We recorded participants' electroencephalogram (EEG) in association with their performance on a cognitive control task (i.e., the Eriksen flanker task).

We set out to test a number of hypotheses, which were all specified in detail on the OSF (https://osf.io/r4k $2 \mathrm{p} /$ registrations). The most prominent hypotheses were the following. (H1, Hypothesis 1) We expected that participants would report enhanced subjective performance in the cognitive enhancement compared with the impairment condition. (H2, hypothesis 2) We expected that participants would attribute errors more often to the brain stimulation device (i.e., externalization of agency) in the impairment condition compared with the enhancement and the control condition. We also expected reduced feelings of agency in the enhancement compared with the control (i.e., no stimulation) condition, because participants would feel less in control over their performance when under the presumed influence of the brain stimulation device compared with when performing the task on their own. (H3, Hypothesis 3) We predicted a stronger ERN in response to errors in the enhancement condition compared with the impairment and the control condition. (H4, Hypothesis 4) We expected a reduced ERN in response to errors in the impairment compared with the enhancement and the control condition. (H5, Hypothesis 5) We expected a relationship between the agency rating and the ERN amplitude in the impairment condition: we hypothesized that a stronger attribution of the error to the brain stimulation device would be associated with a reduced ERN amplitude. These hypotheses provide a direct replication of our earlier study.

Next to this, we also had a moderation hypothesis. (H6, Hypothesis 6) We predicted that the expectancy effects on behavior and the ERN would be stronger for participants that are highly suggestible. Thus, we expected that for participants 
scoring high on the Tellegen Absorption Scale (Tellegen and Atkinson 1974), the effects that were specified above (H1H5) would be enhanced compared with participants scoring low on this scale. ${ }^{1}$

In our preregistered analysis plan, we also specified an additional hypothesis about the neural mechanisms underlying our expectancy manipulation. Specifically, we hypothesized (H7, Hypothesis 7) that expectations about enhanced versus impaired cognitive performance would be accompanied during the induction phase by an increase in theta power over frontal regions - as a proxy for increased cognitive and attentional control (Cavanagh and Frank 2014). That is, we expected that while the placebo tDCS device was "ramping up" its activity during the induction phase, participants would try to benefit from this mental boost in the improvement condition, by trying to increase their attentional focus on the upcoming cognitive task. This would likely be reflected in an enhanced theta power in the enhancement compared with the impairment and the control condition, as, for instance, focused attention has been associated with increased theta power (Baijal and Srinivasan 2010).

We also hypothesized (H8) that during the enhancement compared with the impairment and the control condition, a stronger alpha suppression over occipital sites would be observed in relation to the processing of the stimulus, which could reflect an enhanced process of attentional control (Klimesch 2012). Previous studies have shown for instance that people with a profound experience with mindfulness meditation show an enhanced suppression of alpha power, in association with the processing of tactile and visual stimuli, reflecting an enhanced process of selective attention (Kerr et al. 2011; Kerr et al. 2013). Similarly, we expected that the expectations of cognitive enhancement versus impairment would also affect attentional processes, which might be reflected in a modulation of occipital alpha power.

The study design, hypothesis, and analysis plan including a priori exclusion criteria have been preregistered on the Open Science Framework prior to conducting the study (https://osf. io/f74q3).

\section{Methods}

\section{Participants}

As indicated in our preregistered sampling plan, we aimed to have usable data from 60 participants. Given that we anticipated that EEG data from about $1 / 5$ of all participants would

\footnotetext{
${ }^{1}$ Initially we were planning to include the so-called White Christmas task as an additional measure for suggestibility (Merckelbach and van de Ven 2001), but due to oversight during the setting up of the study, this task was not administered for the actual testing.
}

not be usable for analysis (e.g., due to noisy electrodes, excessive head or eye movements), we collected data from 72 participants in total. Participants were excluded prior to analysis based on visual inspection of the raw EEG data. Participants for whom more than 5 electrodes needed to be interpolated or participants for whom fewer than 5 trials remained within a specific condition after an automated artifact rejection procedure were excluded from analysis. For the final analysis, we included data from 57 participants (mean age $=26.9$ years, $\mathrm{SD}=11.64$, range $=19-69$ years), of which 38 identified as female. Participants were recruited through the university participant recruitment system and through an advertisement in a local newspaper, asking for volunteers to participate in a study to experience the effects of transcranial brain stimulation for cognitive enhancement. The mean level of education was 4.67 $(\mathrm{SD}=.81)$, where 1 corresponds to primary school and 5 to having completed high school or a university degree. The mean level of income was very low, ${ }^{2}$ as many participants were students or participated in response to a newspaper advertisement to earn money by participating in a study. The average perceived subjective socio-economic status (SES) was $71.2(\mathrm{SD}=16.74)$, where 1 referred to the lowest SES and 100 to the highest SES level.

\section{Experimental Setup and Procedure}

For an overview of the experimental setup and procedure, please see Figure 1. Participants were equipped with a real battery-driven Eldtih DC Stimulator (i.e., as used for transcranial direct current stimulation (tDCS); NeuroConn, GmbH, Ilmenau, Germany), but during the actual experiment, we never actually stimulated the brain - as our manipulation relied entirely on verbally induced expectations regarding the potential positive or adverse effects that the device could induce. We used $3 \times 3-\mathrm{cm}$ conductive electrodes, which were placed in saline-soaked sponges and held in place by rubber bands at electrode locations Afz and CPz on top of the electroencephalogram (EEG) cap. Thus, in different blocks, participants were instructed that the stimulation could enhance or decrease their cognitive performance. A "neutral" control condition was included in which participants were instructed that no brain stimulation would be applied. Please note that this was a single-blind study, in which the participants were always unaware of the fact that the tDCS device would never be switched on. The experimenter however was well aware of the fact that the tDCS apparatus was only used to increase the credibility of the verbal suggestion, but was in fact inactive. Both the experimenter and the participant were aware of the verbal suggestions that were used, as the main aim was to maximize expectancy effects.

\footnotetext{
${ }^{2}$ The mean reported income level was $1.51(\mathrm{SD}=1.27)$, where 1 corresponds to a low level of income and 7 to two times the modal income in Dutch society.
} 

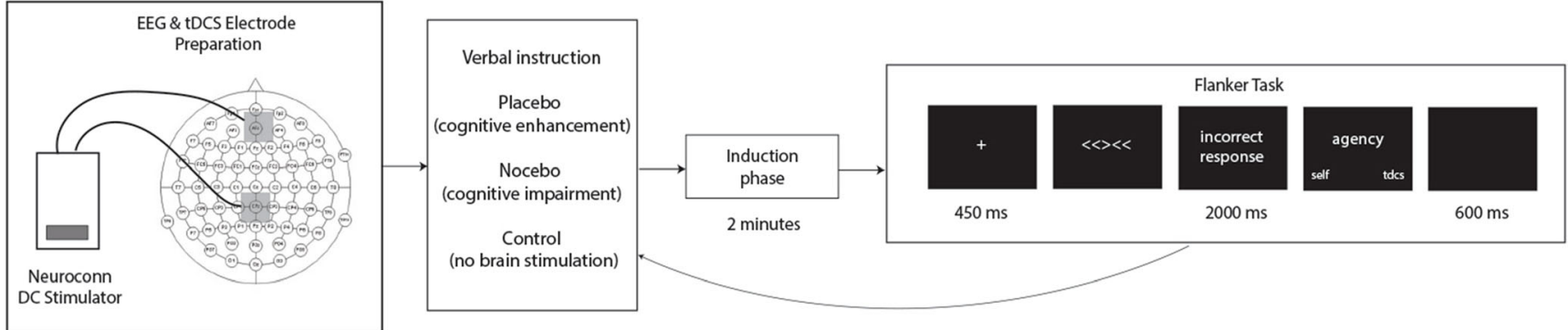

Fig. 1 Experimental setup and procedure. Participants were equipped with the EEG cap and tDCS electrodes. Prior to each block they received verbal instructions regarding the alleged effects of the brain stimulation. This was followed by an induction phase, during which the tDCS was ramped up in the placebo and the nocebo condition in order to increase the credibility of the verbal instruction. Next participants

Participants' EEG was recorded in association with their performance on a cognitive control task (i.e., the Eriksen flanker task). The task was presented on a computer screen $(1920 \times 1080$ pixels; diagonal $60 \mathrm{~cm})$ placed at approximately $40 \mathrm{~cm}$ from the participant's eyes. The task was programmed using Presentation Software (version 19.0; Neurobehavioral Systems, CA, USA). The difficulty of the flanker task was dynamically adjusted based on the participants' performance, in order to allow a comparable behavioral performance between the different blocks. This was accomplished by instructing participants to speed up and respond faster if their performance was higher than $70 \%$ accuracy. The initial response interval for each participant was set at $1000 \mathrm{~ms}$ and this was adjusted dynamically (i.e., increased or decreased by $50 \mathrm{~ms}$ ) based on their performance in the preceding trials throughout each experimental block. There was no absolute minimum or maximum range in which participants should respond, but in practice, the minimum response interval was $300 \mathrm{~ms}$ and the maximum response interval was $1400 \mathrm{~ms}$. The rationale for keeping the behavioral performance constant was that the ERN amplitude is directly related to the likelihood of making an error (Nieuwenhuis et al. 2003). Accordingly, in order to allow a comparison of the ERN amplitudes between the different blocks that is not confounded with the actual number of errors, we decided to keep the performance comparable between blocks.

Each experimental block started with 10 practice trials and consisted of 160 experimental trials which were presented in 8 mini-blocks of 20 trials. Fifty percent of all trials were congruent, in which the central character was pointing in the same direction as the distractors (e.g., " $<<<<<")$ and the other $50 \%$ of trials consisted of incongruent stimuli (e.g., " $<<><<")$. Each trial started with the presentation of a fixation cross for $450 \mathrm{~ms}$, followed by the flanker stimulus, which was presented until the participant responded by pressing a button, or-if the response took too long (see below) - an error message was presented for $2000 \mathrm{~ms}$, encouraging the participant to respond faster on the next trial. If the participant made an incorrect performed the Flanker task as a measure of cognitive control. Their objective performance was kept at a constant accuracy level, by dynamically adjusting the task difficulty. Following each incorrect response on this task participants were required to indicate to what extent they felt the error was caused by themselves or by the brain stimulation. After a short break, the next experimental block was started

response, they were prompted with a screen to indicate their feeling of agency over the error. A black screen was presented for $600 \mathrm{~ms}$ in between different trials.

Following incorrect trials, participants were presented with feedback regarding their performance (i.e., incorrect or too slow). Whenever participants made an incorrect response, they were required to indicate whether they believed the response was influenced by the brain stimulation, by using a visual analog scale, ranging from 1 to $7(1=$ not at all; $7=$ very much). Following each experimental block (i.e., enhancement, impairment, control), participants were asked to rate their subjective perception of their performance. We also asked them to rate the perceived efficacy of the brain stimulation through 3 different questions, i.e., (1) To what extent did you have the feeling that the tDCS affected your performance on the flanker task? (2) To what extent did you feel that your performance on the flanker task was enhanced or impaired through the brain stimulation? (3) To what extent do you think you have a specific sensitivity to use the potential of brain stimulation?), which were answered by using a 5 -point scale $(1=$ not at all $/$ worse; $5=$ very much $/$ better $)$.

We used different ways to boost the expectations of the participants. We used verbal instructions to emphasize the alleged potential of tDCS to boost or impair their performance (i.e., the specific instructions that we presented to participants are included on the OSF, see https://osf.io/r4k2p/files/). We presented participants with a plausible neuroscientific explanation about how electrical brain stimulation could alter the neural energy in their brain at different sites, thereby positively or negatively affecting their performance. Prior to each experimental stimulation block, participants received a short 1-min ramp-up 1-mA anodal stimulation with the tDCS electrodes resulting in a slightly tingling sensation at the skin. This sham stimulation protocal was based on similar stimulation protocols that are typically included as a control condition in the lab at the University of Amsterdam (Reteig et al. 2018; Talsma et al. 2017). Also, as the sham anodal stimulation was the same in both the enhancement and the 
impairment condition, any effect related to direct stimulation of the brain could not be attributed to the tDCS. In association with the ramp-up stimulation, we also used an induction phase of 2 min prior to each block, during which participants were instructed to try to optimally exploit the effects of the tDCS device, by having their eyes closed and by focusing their attention on the brain stimulation. Following the induction phase, the experimental task started and participants performed the flanker task which lasted between 10 and 15 min each block.

\section{Dependent Measures and Analysis}

The main dependent measures consisted of (1) agency ratings over errors, (2) the error-related negativity (i.e., the difference in event-related potential (ERP) amplitude between incorrect and correct trials), and (3) perceived subjective performance. We also assessed the moderating role of individual differences in suggestibility as measured using the "Tellegen Absorption Scale" in relation to our experimental manipulations - as previous studies have shown that these personality traits determine the efficacy of expectancy manipulations (Andersen et al. 2014). The absorption scale consisted of 34 items (e.g., "I can be deeply moved by a sunset"), which were completed using a 4-point scale ( $1=$ not applicable at all, $4=$ applies to a strong extent). At the end of the experiment, we asked participants about their experienced side effects of the brain stimulation by indicating which of the following side effects were experienced during the study: headache, neck ache, muscle spasms (in the neck or face), tingling sensations under tDCS electrodes, burning sensation under tDCS electrodes, uncomfortable feeling (non-specific), which were rated on a 5-point scale $(1=$ not at all, $5=$ very much $)$.

This single-blinded study thus used a within-subjects repeated measures design, including a cognitive enhancement, a cognitive impairment, and a control condition. The order of conditions was counterbalanced across participants. For the analysis of individual differences, a mixed design was used, by including the individual difference measures as covariate. Specifically, for the analysis of the subjective performance and the agency ratings a repeated measures ANOVA was used, including condition (impairment, enhancement, control) as within-subjects factor. As specified on the OSF, we expected a main effect of condition, reflected in enhanced subjective performance in the placebo compared with the nocebo and the control condition (H1) and a stronger external attribution of errors in the nocebo condition compared with the placebo and the control condition (H2). Next to frequentist statistics, we also report a Bayesian analysis of our data where relevant (e.g., by using a Bayesian repeated measures ANOVA), which was conducted by using the open-source software package (JASP 2019). A main advantage of the Bayesian approach is that it allows quantification of evidence in favor of both the null and the alternative hypotheses.

\section{EEG Recording and Analysis}

EEG was recorded at $2048 \mathrm{~Hz}$ using the Active-Two system (BioSemi, Amsterdam, Netherlands), consisting of 64 active electrodes that were placed in an EEG cap according to the standard 10/20 system. The horizontal and vertical EOG was measured by placing electrodes on the outer canthi and above and below the subject's left eye. For the EEG data analysis, bad channels were interpolated.

EEG data was analyzed by focusing on the averaged ERP amplitude per condition (i.e., impairment, enhancement, control) and response (i.e., correct vs. incorrect responses). EEG data was time-locked to the button press response by the participant and segmented from - 100 to $500 \mathrm{~ms}$. A - 100- to 0ms baseline correction was applied. Data was filtered using a low cutoff of $1 \mathrm{~Hz}$ and a high cutoff of $30 \mathrm{~Hz}$. An automated correction of ocular artifacts was conducted by using the method implemented in Brain Vision Analyzer (Gratton \& Coles, 1980). Trials in which the amplitude of one of the electrodes exceeded the +150 or $-150 \mu \mathrm{V}$ were rejected. We calculated the averaged ERPs per condition and participant. An automated peak detection algorithm was applied to electrode FCz searching for the local minimum in the 50- to 150-ms time window following the button press response, as this was the time window in which the ERN is typically found maximal (Gehring et al. 2018).

For the analysis of the ERN, we focused on electrode FCz, which typically displays the maximum ERN amplitude. The data was analyzed using a repeated measures ANOVA including condition (impairment, enhancement, control) and response (correct vs. incorrect) as within-subjects factors. We expected an interaction between condition and response $(\mathrm{H} 3$ and H4). The relation between the ERN and the agency rating in the impairment condition was assessed using a Pearson's correlation analysis (H5). We also tested for the correlations in the enhancement and the control condition, but in those conditions we did not expect a significant correlation.

Individual differences in suggestibility as measured using the Tellegen Absorption Scale were included as covariates in the repeated measures ANOVAs described above (H6). When conducting pairwise comparisons, these analyses were corrected for multiple comparisons, using the Bonferroni correction.

For the analysis of the frontal theta power during the induction phase, we segmented the 2-min induction phase in separate 1 -s epochs (Fig. 1). Following a 0- to 1000-ms baseline correction and an automated artifact rejection procedure, we applied a fast Fourier transformation (FFT) to obtain the power for the different frequency bands. For statistical analysis $(N=51$, due to excessive noise/movements during the 
induction phase, fewer participants were included in this analysis), we used the averaged power from 4 to $8 \mathrm{~Hz}$ across electrodes $\mathrm{FCz}$ and $\mathrm{Fz}$ per condition (H7). For the analysis of the occipital alpha power associated with stimulus processing, we segmented the data time-locked to the onset of the flanker stimuli from -200 to $800 \mathrm{~ms}$. Following a -200 - to $800-\mathrm{ms}$ baseline correction and an automated artifact rejection procedure, we applied a FFT. For statistical analysis $(N=56$ participants were retained for this analysis), we used the averaged alpha power from 8 to $12 \mathrm{~Hz}$ across electrodes PO7 and PO8 per condition (H8).

\section{Results}

\section{Subjective Performance, Feelings of Agency, and Objective Performance}

The results are presented in Figure 2. With respect to subjective performance, as expected (H1), participants indicated a better performance in the placebo compared with the nocebo condition $(t(56)=5.39, p<.001, d=.71)$ (see Fig. 2a). Including absorption as covariate did not result in the expected interaction between condition and absorption (H6) $\left(F_{1,55}=.10, p=.75\right)-$ although a main effect of absorption was found $\left(F_{1,55}=11.97\right.$, $\left.p=.001, \eta^{2}=.18\right)$, reflecting that high absorption participants experienced more impairment in their subjective performance across all conditions (see also below: exploratory analysis).

As hypothesized (H2), analysis of the feeling of agency reflected a main effect of condition $\left(F_{2,114}=37.91, p<.001\right.$, $\left.\eta^{2}=.399\right)$. Post hoc $t$ tests indicated that participants felt more agency in the placebo compared with the nocebo condition $(t(57)=4.72, p<.001, d=.62)$; in the control compared with the placebo condition $(t(57)=-4.50, p<.001, d=.59)$; and in the control compared with the nocebo condition $(t(57)=-$ $7.89, p<.001, d=-1.04$ ) (see Fig. 2a). Contrary to the moderation hypothesis (H6), including absorption as an additional covariate in the analysis did not reveal the hypothesized interaction between condition and absorption $\left(F_{2,112}=.12\right.$, $p=.89$ ).

Analysis of the accuracy indicated that participants made more incorrect responses in response to incongruent compared a

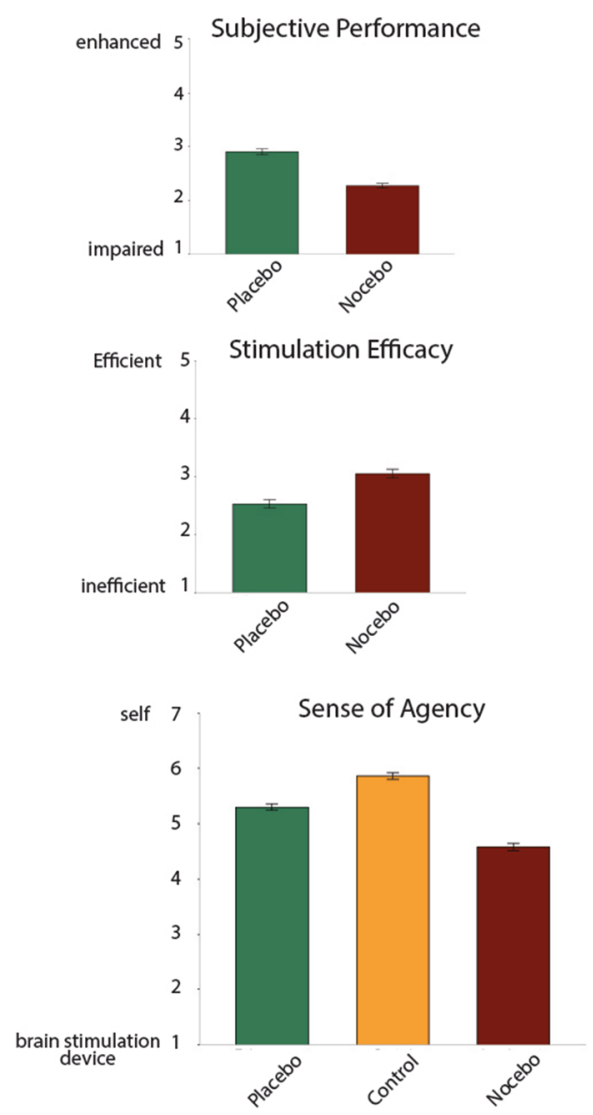

Fig. 2 A. Subjective performance (top panel), perceived stimulation efficacy (middle panel) and sense of agency (lower panel) for the placebo (green bars), nocebo (red bars) and the control condition (yellow bars). B. Event-related potentials time-locked to response-onset b
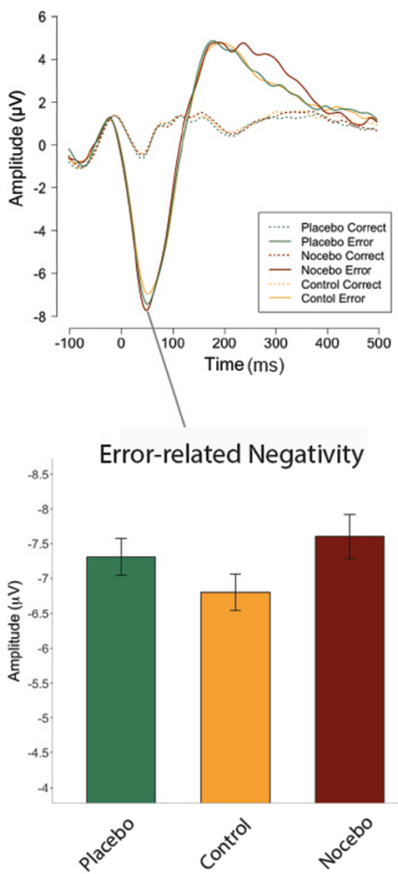

C Correlation Placebo Condition
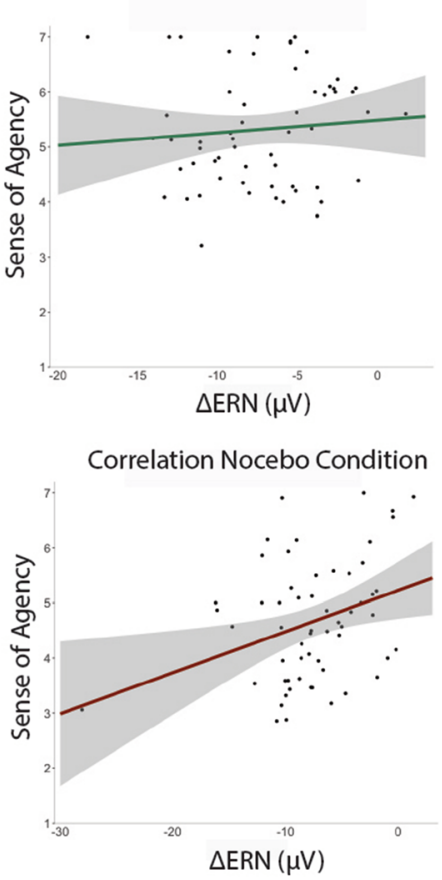

for the different experimental conditions (top panel) and the error-related negativity (ERN) amplitude as a function of condition (lower panel). C. Correlations between the ERN amplitude and sense of agency for the placebo condition (upper panel) and the nocebo condition (lower panel) 
Table 1 Accuracy (proportion of errors) and reaction times (in milliseconds) as a function of experimental condition and stimulus condition. Standard deviations are between parentheses

\begin{tabular}{llll}
\hline & & Proportion of errors & Reaction times \\
\hline Placebo & Congruent & $.00(.01)$ & $370.0(54.8)$ \\
& Incongruent & $.11(.05)$ & $422.6(55.8)$ \\
Nocebo & Congruent & $.01(.01)$ & $362.8(46.4)$ \\
& Incongruent & $.12(.05)$ & $416.2(49.4)$ \\
Control & Congruent & $.01(.01)$ & $363.2(47.6)$ \\
& Incongruent & $.12(.05)$ & $414.6(53.3)$ \\
\hline
\end{tabular}

with congruent trials $\left(F_{1,57}=418.12, p<.001, \eta^{2}=.880\right)$ (see Table 1). Accuracy did not differ between conditions $(F=.94$, $p=.395)$. Analysis of the reaction times indicated that participants responded slower to incongruent compared with congruent trials $\left(F_{1,57}=846.95, p<.001, \eta^{2}=.938\right)($ see Table 1$)$. As expected, the reaction times did not differ between conditions $(F<1.45, p=.239)$.

\section{ERN Analysis}

For the analysis of the ERN amplitudes, a main effect of response $\left(F_{1,57}=245.82, p<.001, \eta^{2}=.81\right)$ reflected a stronger ERN amplitude for incorrect compared with correct responses (see Fig. 2b). However, contrary to our predictions (H3 and $\mathrm{H} 4)$, the effect of condition and the interaction between condition and response were not significant $(F=1.06, p=.349)$. A Bayesian analysis indicated that the data was 20 times less likely under the model including response and condition as main factors than under the model including only response as a factor $\left(\mathrm{BF}_{01}=20.23\right)$; the data was about 200 times less likely under the model including both main effects and the interaction between condition and response compared with the model including only response as a factor $\left(\mathrm{BF}_{01}=\right.$ 211.96). This indicates strong evidence that the ERN amplitude was not affected by the expectancy manipulation.
Table 3 Theta power $(4-8 \mathrm{~Hz})$ and alpha power $(8-12 \mathrm{~Hz})$ over frontal and occipital electrodes for the different experimental conditions. Standard deviations are between parentheses

\begin{tabular}{llllll}
\hline & \multicolumn{3}{l}{ Theta power } & & \multicolumn{2}{l}{ Alpha power } \\
\cline { 2 - 3 } \cline { 6 - 6 } & FCz & Fz & & PO7 & PO8 \\
\hline Placebo & $1.20(.81)$ & $.94(.70)$ & & $1.94(2.20)$ & $1.99(2.46)$ \\
Nocebo & $1.20(.81)$ & $1.80(1.32)$ & & $1.96(2.25)$ & $1.94(1.89)$ \\
Control & $1.55(.71)$ & $.99(1.09)$ & & $1.91(1.74)$ & $1.98(1.90)$ \\
\hline
\end{tabular}

Including absorption as a covariate in the analysis (H6) did not result in the expected interaction effect between condition and response $\left(F_{2,112}=1.36, p=.262\right)$. Again a Bayesian analysis indicated that the data was four times more likely under a model without absorption as a covariate $\left(\mathrm{BF}_{01}=3.96\right)$.

Furthermore, we conducted a confirmatory analysis to assess the relationship between the ERN amplitude and feelings of agency (H5). In the placebo condition, the correlation between the ERN and agency was non-significant $(r=.09$, $p=.50$ ) (Table 2). In the nocebo condition, a positive correlation was observed $(r=.33, p=.012)$, reflecting that reduced feelings of agency were associated with a stronger ERN amplitude (see Fig. 2c and Table 4). Note that this effect was in the opposite direction than hypothesized (H5).

\section{Theta and Alpha Power}

Analysis of the theta power including data from both electrode locations during the induction phase prior to the experimental task revealed a main effect of condition $\left(F_{2,100}=8.28\right.$, $\left.p<.001, \eta^{2}=.142\right)$ and an interaction between condition and electrode $\left(F_{2,100}=12.12, p<.001, \eta^{2}=.195\right)$. No significant interaction with electrode $(\mathrm{FCz}, \mathrm{Fz})$ was observed $(F<1)$. Contrary to our predictions (H7), as can be seen in Table 3 , theta power was lower in the placebo compared with the control condition $(t(51)=4.20, p<.001, d=.58)$ and compared

Table 2 Correlations between ERN amplitude, the perceived efficacy, and feelings of agency in the different experimental conditions

\begin{tabular}{|c|c|c|c|c|c|c|c|}
\hline & ERN placebo & ERN nocebo & ERN control & Efficacy placebo & Efficacy nocebo & Agency placebo & Agency nocebo \\
\hline ERN placebo & - & & & & & & \\
\hline ERN nocebo & $0.451 * * *$ & - & & & & & \\
\hline ERN control & $0.696 * * *$ & $0.441 * * *$ & - & & & & \\
\hline Efficacy placebo & -0.129 & -0.245 & -0.007 & - & & & \\
\hline Efficacy nocebo & -0.112 & $-0.322 *$ & -0.047 & $0.334 *$ & - & & \\
\hline Agency placebo & 0.090 & 0.244 & -0.152 & $-0.643^{* * *}$ & -0.198 & - & \\
\hline Agency nocebo & 0.057 & $0.328 *$ & 0.061 & $-0.454 * * *$ & $-0.612 * * *$ & $0.503 * * *$ & - \\
\hline Agency control & 0.083 & -0.049 & 0.037 & $-0.406^{* *}$ & -0.043 & $0.421 * * *$ & $0.273 *$ \\
\hline
\end{tabular}

$* p<.05, * * p<.01, * * * p<.001$ 
Table 4 Correlations between subjective measures. Abs, absorption; Sub, subjective performance; Eff, perceived efficacy of the brain stimulation

\begin{tabular}{lllllll}
\hline & Abs & Sub placebo & Sub nocebo & Eff placebo & Eff nocebo & Side effects \\
\hline Abs & - & & & & \\
Sub placebo & $-0.242^{*}$ & - & & & \\
Sub nocebo & $-0.400^{* *}$ & 0.192 & - & & \\
Eff placebo & 0.020 & 0.054 & -0.120 & - & - & \\
Eff nocebo & $0.386^{* *}$ & 0.002 & $-0.630^{* * *}$ & $0.334^{*}$ & -0.064 & - \\
Side effects & 0.025 & -0.124 & 0.044 & $0.393^{* *}$ & - \\
\hline
\end{tabular}

$* p<.05, * * p<.01, * * * p<.001$ with the nocebo condition $(t(50)=2.02, p=.049, d=.28)$. Theta power was higher in the nocebo compared with the control condition $(t(51)=2.43, p=.019, d=.34)$. Contrary to our hypothesis (H8), analysis of the stimulus-locked alpha power did not reveal significant differences between conditions $\left(F_{1,55}=.09, p=.92\right)$.

\section{Exploratory Analysis}

The correlations between the different self-report measures are presented in Table 4. As can be seen, high absorption participants felt that their subjective performance was actually hampered by the brain stimulation device - both in the placebo and in the nocebo condition. Also, high absorption participants rated the effectiveness of the device as higher in the nocebo condition. Reported side effects in turn were positively correlated to the perceived efficacy of the device in the placebo condition.

When assessing the correlation between the theta power (which was measured during the pre-experimental induction phase) and the ERN amplitude, we found across all conditions a significant negative relationship between theta power and ERN amplitude (see Table 4). This reflects that participants who had a high theta power during the induction phase were also characterized by a stronger ERN on the subsequent Eriksen flanker task (Table 5).

Following the observation that the perceived efficacy was strongly related to subjectively felt improvement of performance, in an exploratory analysis, we included the perceived efficacy as an additional covariate in the analyses. For the agency ratings, including efficacy as a covariate rendered the main effect of condition mute $\left(F_{2,112}=.03, p=.97\right) .^{3} \mathrm{~A}$ significant interaction between condition and efficacy was found $\left(F_{2,112}=3.51, p=.033, \eta^{2}=.06\right)$, and the main effect of efficacy was significant $\left(F_{1,56}=5.31, p=.025, \eta^{2}=.09\right)$. Overall, participants who more strongly believed in the efficacy of the brain stimulation had lower feelings of agency than people who did not believe in the efficacy (see Fig. 3a). Agency

\footnotetext{
${ }^{3}$ For the ANOVAs, we included the combined efficacy rating across both conditions as covariate in the analysis. For the post hoc tests, we used the efficacy rating separately for each condition.
}

ratings were specifically related to perceived efficacy in the nocebo condition $(r=-.38, p=.003)$, while there was no consistent relation in the placebo condition $(r=-.245$, $p=.064)$ and the control condition $(r=-.034, p=.798)$.

For the analysis of the ERN, we observed a significant interaction between condition and efficacy $\left(F_{2,112}=3.71\right.$, $\left.p=.028, \eta^{2}=.06\right)$, an interaction between response and efficacy $\left(F_{1,56}=6.39, p=.014, \eta^{2}=.10\right)$, and a three-way interaction between condition, response, and efficacy $\left(F_{2,112}=\right.$ $\left.8.92, p<.001, \eta^{2}=.124\right)$. Post hoc analyses indicated that this effect was driven by the nocebo condition: participants who rated the efficacy as high had a stronger ERN than low efficacy participants $(r=-.322, p=.015)$ (see Fig. 3b and Table 2). The ERN did not differ as a function of efficacy for the placebo condition $(r=-.13, p=.334)$. As can be seen in Fig. $3 b$ in the nocebo condition, there was one participant with an extremely strong ERN amplitude. ${ }^{4}$ When excluding this outlier, the correlation between the ERN and the efficacy was no longer significant $(r=-.26, p=.053)$.

\section{Discussion}

Our expectancy manipulation regarding enhanced versus impaired cognitive performance through transcranial stimulation affected the perceived subjective performance and feelings of agency. Participants reported impaired performance and reduced feelings of agency in the nocebo condition compared with the placebo and the control condition. These findings are in line with previous studies showing that suggestions regarding cognitive enhancement or impairment can affect feelings of agency and subjective performance on a cognitive control task (Hoogeveen et al. 2018; Schwarz and Buchel 2015; Swiney and Sousa 2013).

\footnotetext{
${ }^{4}$ As we did not specify extreme ERN amplitudes as an a priori exclusion criterion in our pre-registered analysis plan, we decided to keep this participant in all the main analyses that we conducted. Please note that in/exclusion of these participants only affected the observed relation between agency ratings and the ERN amplitude in the nocebo condition. The other observed effects were robust against exclusion of this outlier.
} 
Table 5 Correlations between theta power and ERN amplitudes across the different experimental conditions

\begin{tabular}{lllllll}
\hline & $\theta$ placebo & $\theta$ nocebo & $\theta$ control & ERN placebo & ERN nocebo & ERN control \\
\hline$\Theta$ placebo & - & & & & \\
$\Theta$ nocebo & $0.807^{* * *}$ & - & & & \\
$\Theta$ control & $0.834 * * *$ & $0.795^{* * *}$ & - & & \\
ERN placebo & $-0.341^{*}$ & $-0.386^{* *}$ & $-0.355^{* *}$ & - & - \\
ERN nocebo & $-0.335^{*}$ & $-0.387^{* *}$ & $-0.299^{*}$ & $0.451^{* * *}$ & - \\
ERN control & $-0.313^{*}$ & $-0.382^{* *}$ & $-0.310^{*}$ & $0.696^{* * *}$ & $0.441^{* * *}$ & - \\
\hline
\end{tabular}

$* p<.05, * * p<.01, * * * p<.001$
However, contrary to our hypothesis that the error-related negativity (ERN) would be stronger in the placebo condition - as a marker of surprise in response to unfulfilled expectations (cf., Hoogeveen et al. 2018), we did not find an effect of prior expectations on the ERN amplitude. We found that a stronger ERN was associated with reduced feelings of agency in the nocebo condition. We also observed that participants who rated the perceived efficacy of the tDCS as high had a stronger ERN than participants who rated the efficacy as low in the nocebo condition. We note however that the directionality of these effects was not predicted a priori and that the effects were strongly driven by an extreme outlier. We also note that the current findings appear in direct contrast with our
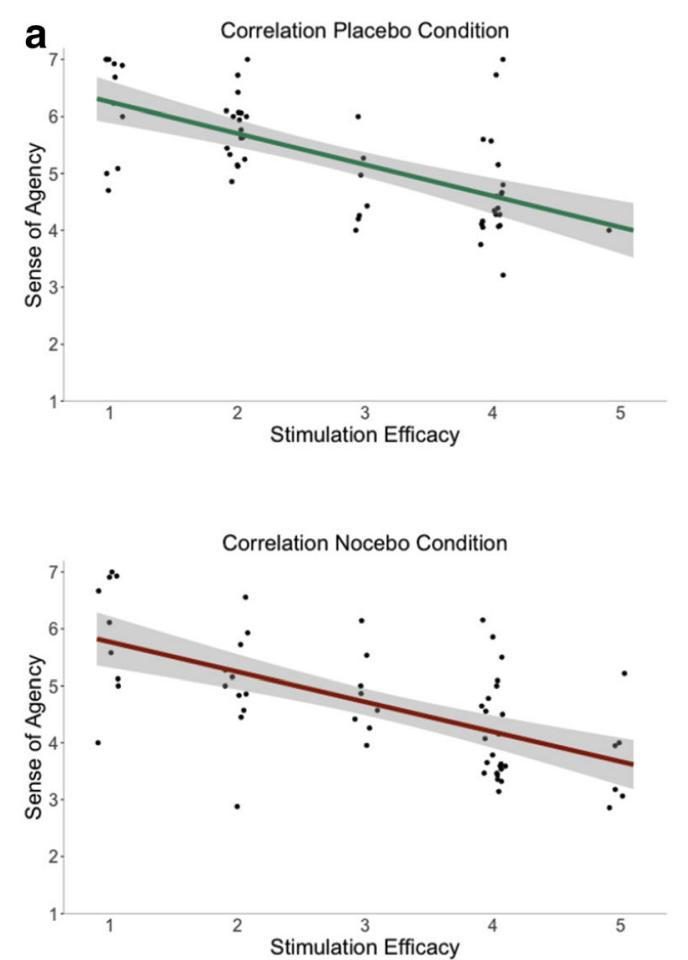

Fig. 3 A. Correlation between perceived efficacy of the stimulation and sense of agency for the placebo condition (upper panel) and the nocebo condition (lower panel). B. Correlation between the ERN amplitude and earlier study in which we found a reverse relationship between feelings of agency and ERN amplitude (Hoogeveen et al. 2018), thus underlining the need for conducting more highpowered studies - especially when it comes to assessing individual differences in cognitive neuroscience research. In our study, participants only felt slightly improved in the placebo condition - scoring around the midpoint of a scale measuring improved versus impaired subjective performance. These findings suggest that overall the nocebo effect in our study was stronger than the placebo effect. This might be related to the fact that we kept participants' objective performance at a constant - relatively low-level, by dynamically changing the response interval. Also, the initial ramping up of the
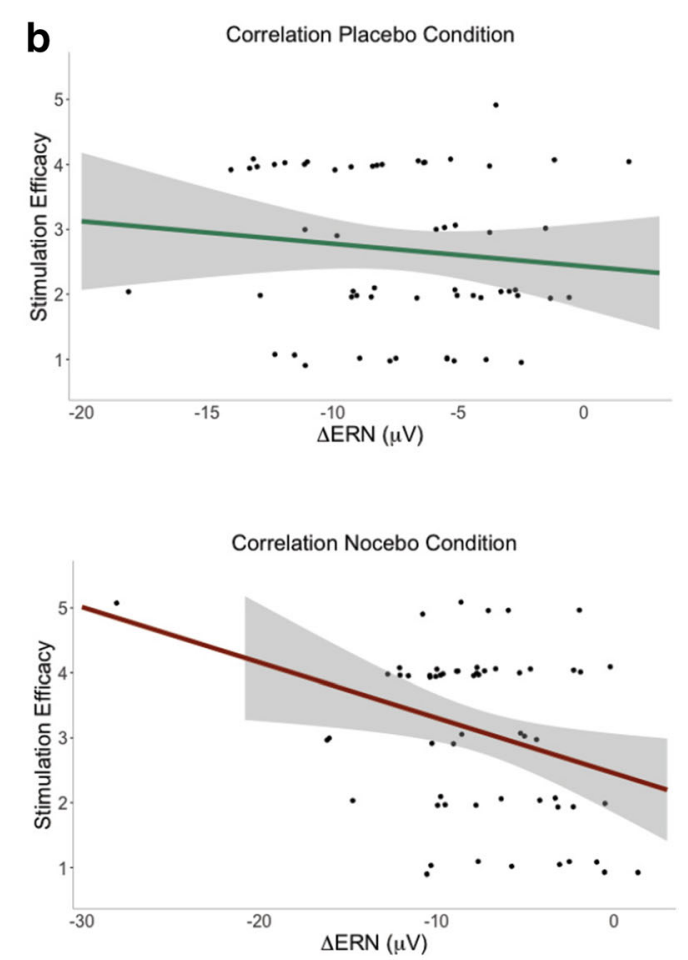

perceived stimulation efficacy for the placebo condition (upper panel) and the nocebo condition (lower panel) 
tDCS might have contributed to the nocebo effect, as many participants experienced the transcranial stimulation as unpleasant, which could have increased their doubts about the potential beneficial effects of the stimulation for their performance.

Whereas some previous studies on placebo cognitive enhancement have reported effects on objective performance (Colagiuri et al. 2011; da Gama et al. 2013; Rozenkrantz et al. 2017), other studies have shown effects only on subjective ratings but not on objective or behavioral measures (Looby and Earleywine 2011; Schwarz and Buchel 2015). The absence of an overall effect of our expectancy manipulation on the ERN in the present study could be related to different factors. First, our verbal instructions may have been insufficient to instill strong and robust expectations. Including a conditioning procedure may be necessary - as is often done in research on placebo analgesia (Schafer et al. 2015). In terms of boosting expectations about cognitive enhancement, through conditioning participants could actually experience enhanced compared with impaired performance during the initial phase of the task, which further reinforces their belief in the potential of the brain stimulation device. Relatedly, the flanker task employed in this study elicited a direct and clear error-related signal accompanying incorrect responses-leaving little ambiguity about the correctness of the participants' performance. A more ambiguous task in which the correctness of the outcomes is less transparent would probably allow stronger top-down effects of participants' prior expectations. Also, repeatedly administering the same task to participants under different conditions provided them with a direct opportunity to compare their performance with the preceding blocks, which could have worked against our verbally induced expectations. Thus, future studies on placebo effects in cognitive enhancement should incorporate stronger experimental inductions and measures that afford sufficient ambiguity in the attribution of erroneous response to the brain stimulation device.

During the induction phase, we found that theta power was enhanced for the nocebo condition compared with the placebo and the control condition. During the placebo condition, in contrast, theta power was lower compared with the other conditions. This finding was contrary to our expectations, as we hypothesized that theta power would be increased during the placebo condition as a proxy for increased cognitive control (Cavanagh and Frank 2014). We can only speculate about the potential psychological mechanism underlying this unexpected finding. It could reflect an ironic effect: participants may have been especially motivated to counter any potential adverse effects of the stimulation during the nocebo condition, while the placebo condition did not require an active mindset on their behalf (i.e., as the brain stimulation device would already improve their performance). In previous studies using placebo brain stimulation, we have observed similar ironic effects, which could arise due to the obvious lack of double-blind experimental procedures, thereby triggering participants' suspicion (van Elk 2015). We also found a consistent relation between theta power during the induction phase and the ERN amplitude during the experimental task, suggesting that both measures tap into a similar underlying neurocognitive construct. This is in line with meta-analytic studies suggesting a common neural origin of theta and ERN responses, which could reflect individual differences in frontal-midline signals related to domain-general cognitive control processes (Cavanagh and Shackman 2015).

At a subjective level, we observed that participants scoring high on the absorption scale felt more hampered by the brain stimulation device, both in the placebo and in the nocebo condition. High absorption participants also rated the efficacy of the brain stimulation as higher in the nocebo condition. This is in line with another study, showing that participants scoring high on selftranscendence - a trait that is strongly related to absorptionwere most responsive to the alleged effects of different types of lighting on cognitive performance (Fresson et al. 2017). We note that the effects of absorption on perceived efficacy could partly reflect demand characteristics, as high absorption participants may have an implicit motivated tendency to answer in a socially desirable way (Lifshitz et al. 2019). The relation between reported side effects and belief in the efficacy of the brain stimulation is reminiscent of research on placebo effects on anti-depressants: in double-blind placebo-controlled studies, about $80 \%$ of the participants correctly guess their condition, based on physiological side effects (i.e., a problem that is known as "breaking blind"; cf., Rabkin et al. 1986). The reported side effects, such as increased sweating and a dry mouth, in turn underlie self-reported improvement of depressive symptoms, because they reinforce the patient's belief in the efficacy of the medication (Kirsch 2009). It has even been shown that also in conventional tDCS research participants often break blind regarding their experimental condition, which in turn could increase the expectancy effects (Turi et al. 2019).

In transcranial stimulation research, there is an ongoing discussion about the efficacy of neural stimulation techniques, such as tDCS, and their potential to affect objective markers of performance. Critics point out the apparently limited efficacy of tDCS to actually penetrate the skull and to up- or downregulate the underlying neural tissue (Horvath et al. 2014; Horvath et al. 2015). Proponents of brain stimulation techniques argue that more refined stimulation protocols may be necessary, taking into account individual variability in brain structure and function for instance (Antal et al. 2015; Opitz et al. 2015). Expectancy effects could partly underlie the perceived efficacy of tDCS effects (Dawood et al. 2019; Turi et al. 2019) and the present study demonstrates that expectations about cognitive enhancement versus impairment clearly exert subjective but not objective effects. In our study design, we kept the accuracy at a constant level by adjusting the time window for responding - but even then the response times turned out to be comparable between the different conditions. Our findings and the lack of expected transcranial stimulation on neural markers of error processing are in line with a recent study in 
which expectations and tDCS stimulation were independently manipulated, showing that cognitive and motor performance was not affected, neither by expectations nor by real versus sham tDCS (Rabipour et al. 2019). Thus, a challenge for future studies on transcranial stimulation is to determine the potential additive effects of prior expectations and actual brain stimulation, and the boundary conditions of these effects in terms of comparing subjective and objective performance.

\section{Conclusions}

People's fascination for neuroscience and their belief in enhancement through transcranial stimulation offer intriguing possibilities for understanding the psychological and neurocognitive mechanisms underlying similar phenomena, in which people are enchanted by an external power (e.g., traditional beliefs in magic and supernatural agents in religious worldviews). It also provides direct insight into the role of expectancy effects in neurofeedback and neurostimulation experiments (Schabus et al. 2017). The present study shows that expectations about cognitive enhancement versus impairment affected subjective feelings of performance on a cognitive control task and resulted in an externalization of errors to the brain stimulation device, though we found no evidence that it affected neural responses to errors.

\section{Compliance with Ethical Standards}

All procedures performed in this study involving human participants were in accordance with the ethical standards of the institutional research committee (application no. 2018-SP-9064; https://www.lab.uva.nl/lab/ethics/) and with the 1964 Helsinki declaration and its later amendments or comparable ethical standards. Informed consent was obtained from all individual participants included in the study. This study was supported by a grant from the BIAL Foundation on placebo brain stimulation (grant no. 076/2016). The authors declare that they have no conflict of interest.

Open Access This article is licensed under a Creative Commons Attribution 4.0 International License, which permits use, sharing, adaptation, distribution and reproduction in any medium or format, as long as you give appropriate credit to the original author(s) and the source, provide a link to the Creative Commons licence, and indicate if changes were made. The images or other third party material in this article are included in the article's Creative Commons licence, unless indicated otherwise in a credit line to the material. If material is not included in the article's Creative Commons licence and your intended use is not permitted by statutory regulation or exceeds the permitted use, you will need to obtain permission directly from the copyright holder. To view a copy of this licence, visit http://creativecommons.org/licenses/by/4.0/.

\section{References}

Ali, S. S., Lifshitz, M., \& Raz, A. (2014). Empirical neuroenchantment: from reading minds to thinking critically. Frontiers in Human Neuroscience, 8, 357. https://doi.org/10.3389/fnhum.2014.00357.
Andersen, M., Schjødt, U., Nielbo, K. L., \& Sørensen, J. (2014). Mystical experience in the lab. Method and Theory in the Study of Religion, 26, 217-245.

Antal, A., Keeser, D., Priori, A., Padberg, F., \& Nitsche, M. A. (2015). Conceptual and procedural shortcomings of the systematic review "evidence that transcranial direct current stimulation (tDCS) generates little-to-no reliable neurophysiologic effect beyond MEP amplitude modulation in healthy human subjects: a systematic review" by Horvath and co-workers. Brain Stimulation: Basic, Translational, and Clinical Research in Neuromodulation, 8(4), 846-849.

Baijal, S., \& Srinivasan, N. (2010). Theta activity and meditative states: spectral changes during concentrative meditation. Cognitive Processing, 11(1), 31-38. https://doi.org/10.1007/s10339-0090272-0.

Benedetti, F., Carlino, E., \& Pollo, A. (2011). How placebos change the patient's brain. Neuropsychopharmacology, 36(1), 339-354. https:// doi.org/10.1038/npp.2010.81.

Cavanagh, J. F., \& Frank, M. J. (2014). Frontal theta as a mechanism for cognitive control. Trends in Cognitive Sciences, 18(8), 414-421. https://doi.org/10.1016/j.tics.2014.04.012.

Cavanagh, J. F., \& Shackman, A. J. (2015). Frontal midline theta reflects anxiety and cognitive control: meta-analytic evidence. Journal of Physiology-Paris, 109(1-3), 3-15. https://doi.org/10.1016/j. jphysparis.2014.04.003.

Colagiuri, B., Livesey, E. J., \& Harris, J. A. (2011). Can expectancies produce placebo effects for implicit learning? Psychonomic Bulletin \& Review, 18(2), 399-405. https://doi.org/10.3758/s13423-0100041-1.

Colloca, L., \& Finniss, D. (2012). Nocebo effects, patient-clinician communication, and therapeutic outcomes. Jama-Journal of the American Medical Association, 307(6), 567-568. https://doi.org/ 10.1001/jama.2012.115.

da Gama, P. A. M. D., Slama, H., Caspar, E. A., Gevers, W., \& Cleeremans, A. (2013). Placebo-suggestion modulates conflict resolution in the Stroop Task. Plos One, 8(10), e75701. https://doi.org/ 10.1371/journal.pone.0075701.

Dawood, A. B., Dickinson, A., Aytemur, A., Howarth, C., Milne, E., \& Jones, M. (2019). Investigating the effects of tDCS on visual orientation discrimination task performance:"the possible influence of placebo". Journal of Cognitive Enhancement, 1-15.

Falkenstein, M., Hohnsbein, J., Hoormann, J., \& Blanke, L. (1991). Effects of crossmodal divided attention on late ERP components. II. Error processing in choice reaction tasks. Electroencephalography and Clinical Neurophysiology, 78(6), 447-455. https://doi.org/10.1016/0013-4694(91)90062-9.

Farah, M. J. (2002). Emerging ethical issues in neuroscience. Nature Neuroscience, 5(11), 1123-1129.

Figee, M., Luigjes, J., Smolders, R., Valencia-Alfonso, C. E., van Wingen, G., de Kwaasteniet, B., et al. (2013). Deep brain stimulation restores frontostriatal network activity in obsessive-compulsive disorder. Nature Neuroscience, 16(4), 386-387. https://doi.org/10. 1038/nn.3344.

Fresson, M., Dardenne, B., Geurten, M., Anzaldi, L., \& Meulemans, T. (2017). The role of self-transcendence and cognitive processes in the response expectancy effect. Psychologica Belgica, 57(2), 77-92. https://doi.org/10.5334/pb.364.

Gehring, W. J., Goss, B., Coles, M. G. H., Meyer, D. E., \& Donchin, E. (1993). A neural system for error-detection and compensation. Psychological Science, 4(6), 385-390. https://doi.org/10.1111/j. 1467-9280.1993.tb00586.x.

Gehring, W. J., Goss, B., Coles, M. G., Meyer, D. E., \& Donchin, E. (2018). The error-related negativity. Perspectives on Psychological Science, 13(2), 200-204.

Hoogeveen, S., Schjoedt, U., \& van Elk, M. (2018). Did I do that? Expectancy effects of brain stimulation on error-related negativity 
and sense of agency. Journal of Cognitive Neuroscience, 30(11), 1720-1733. https://doi.org/10.1162/jocn_a 01297.

Horvath, J. C., Carter, O., \& Forte, J. D. (2014). Transcranial direct current stimulation: five important issues we aren't discussing (but probably should be). Frontiers in Systems Neuroscience, 8(2).

Horvath, J. C., Forte, J. D., \& Carter, O. (2015). Evidence that transcranial direct current stimulation (tDCS) generates little-to-no reliable neurophysiologic effect beyond MEP amplitude modulation in healthy human subjects: a systematic review. Neuropsychologia, 65, 213236.

JASP (Version 0.11.1) (Version 0.11.1). (2019): JASP Team. Retrieved from https://jasp-stats.org/

Kerr, C. E., Jones, S. R., Wan, Q., Pritchett, D. L., Wasserman, R. H., Wexler, A., et al. (2011). Effects of mindfulness meditation training on anticipatory alpha modulation in primary somatosensory cortex. Brain Research Bulletin, 85(3-4), 96-103. https://doi.org/10.1016/j. brainresbull.2011.03.026.

Kerr, C. E., Sacchet, M. D., Lazar, S. W., Moore, C. I., \& Jones, S. R. (2013). Mindfulness starts with the body: somatosensory attention and top-down modulation of cortical alpha rhythms in mindfulness meditation. Frontiers in Human Neuroscience, 7, 12. https://doi.org/ 10.3389/fnhum.2013.00012.

Kirsch, I. (2009). Antidepressants and the placebo response. Epidemiology and Psychiatric Sciences, 18(4), 318-322.

Klimesch, W. (2012). alpha-band oscillations, attention, and controlled access to stored information. Trends in Cognitive Sciences, 16(12), 606-617. https://doi.org/10.1016/j.tics.2012.10.007.

Lifshitz, M., van Elk, M., \& Luhrmann, T. M. (2019). Absorption and spiritual experience: a review of evidence and potential mechanisms. Consciousness and Cognition, 73, 102760. https://doi.org/10.1016/ j.concog.2019.05.008.

Looby, A., \& Earleywine, M. (2011). Expectation to receive methylphenidate enhances subjective arousal but not cognitive performance. Experimental and Clinical Psychopharmacology, 19(6), 434-445. https://doi.org/10.1037/a0025252.

Maij, D. L. R., \& van Elk, M. (2018). Getting absorbed in experimentally induced extraordinary experiences: effects of placebo brain stimulation on agency detection. Consciousness and Cognition, 66, 1-16. https://doi.org/10.1016/j.concog.2018.09.010.

Maij, D. L. R., van Elk, M., \& Schjoedt, U. (2019). The role of alcohol in expectancy-driven mystical experiences: a pre-registered field study using placebo brain stimulation. Religion Brain \& Behavior, 9(2), 108-125. https://doi.org/10.1080/2153599x.2017.1403952.

Meissner, K., Fässler, M., Rücker, G., Kleijnen, J., Hróbjartsson, A., Schneider, A., \& Linde, K. (2013). Differential effectiveness of placebo treatments: a systematic review of migraine prophylaxis. JAMA Internal Medicine, 173(21), 1941-1951.

Merckelbach, H., \& van de Ven, V. (2001). Another white christmas: fantasy proneness and reports of 'hallucinatory experiences' in undergraduate students. Journal of Behavior Therapy and Experimental Psychiatry, 32(3), 137-144.

Nieuwenhuis, S., Yeung, N., van den Wildenberg, W., \& Ridderinkhof, K. R. (2003). Electrophysiological correlates of anterior cingulate function in a go/no-go task: effects of response conflict and trial type frequency. Cognitive Affective \& Behavioral Neuroscience, 3(1), 17-26. https://doi.org/10.3758/Cabn.3.1.17.

O'Connor, C., Rees, G., \& Joffe, H. (2012). Neuroscience in the public sphere. Neuron, 74(2), 220-226.

Opitz, A., Paulus, W., Will, S., Antunes, A., \& Thielscher, A. (2015). Determinants of the electric field during transcranial direct current stimulation. Neuroimage, 109, 140-150.

Rabipour, S., Vidjen, P. S., Remaud, A., Davidson, P. S., \& Tremblay, F. (2019). Examining the interactions between expectations and tDCS effects on motor and cognitive performance. Frontiers in Neuroscience, 12(999), 1-12.
Rabkin, J. G., Markowitz, J. S., Stewart, J., Mcgrath, P., Harrison, W., Quitkin, F. M., \& Klein, D. F. (1986). How blind is blind - assessment of patient and doctor medication guesses in a placebocontrolled trial of imipramine and Phenelzine. Psychiatry Research, 19(1), 75-86. https://doi.org/10.1016/0165-1781(86) 90094-6.

Racine, E., Bell, E., \& Illes, J. (2008). Can we read minds? Ethical challenges and responsibilities in the use of neuroimaging research. In J. Gioradano \& B. Gorijn (Eds.), Scientific, philosophical and ethical perspectives in neuroethics (pp. 246-270). Cambridge: Cambridge University Press.

Reteig, L. C., Knapen, T., Roelofs, F. J. F. W., Ridderinkhof, K. R., \& Slagter, H. A. (2018). No evidence that frontal eye field tDCS affects latency or accuracy of prosaccades. Frontiers in Neuroscience, 12, 617. https://doi.org/10.3389/fnins.2018.00617.

Roskies, A. (2006). Neuroscientific challenges to free will and responsibility. Trends in Cognitive Sciences, 10(9), 419-423.

Rozenkrantz, L., Mayo, A. E., Ilan, T., Hart, Y., Noy, L., \& Alon, U. (2017). Placebo can enhance creativity. Plos One, 12(9), e0182466. https://doi.org/10.1371/journal.pone.0182466.

Schabus, M., Griessenberger, H., Gnjezda, M. T., Heib, D. P. J., Wislowska, M., \& Hoedlmoser, K. (2017). Better than sham? A double-blind placebo-controlled neurofeedback study in primary insomnia. Brain, 140, 1041-1052. https://doi.org/10.1093/brain/ awx011.

Schafer, S. M., Colloca, L., \& Wager, T. D. (2015). Conditioned placebo analgesia persists when subjects know they are receiving a placebo. Journal of Pain, 16(5), 412-420. https://doi.org/10.1016/j.jpain. 2014.12.008.

Schwarz, K. A., \& Buchel, C. (2015). Cognition and the placebo effect dissociating subjective perception and actual performance. Plos One, 10(7), e0130492. https://doi.org/10.1371/journal.pone. 0130492.

Slaby, J., \& Choudhury, S. (2011). Proposal for a critical neuroscience. In S. Choudhury \& J. Slaby (Eds.), Critical neuroscience: a handbook of the social and cultural contexts of neuroscience (pp. 29-52). Oxford: Blacwell Publishing.

Steenbergen, L., Sellaro, R., Hommel, B., Lindenberger, U., Kühn, S., \& Colzato, L. S. (2016). "Unfocus" on foc. us: commercial tDCS headset impairs working memory. Experimental Brain Research, 234(3), 637-643.

Swiney, L., \& Sousa, P. (2013). When our thoughts are not our own: investigating agency misattributions using the Mind-to-Mind paradigm. Consciousness and Cognition, 22(2), 589-602.

Talsma, L. J., Kroese, H. A., \& Slagter, H. A. (2017). Boosting cognition: effects of multiple-session transcranial direct current stimulation on working memory. Journal of Cognitive Neuroscience, 29(4), 755768. https://doi.org/10.1162/jocn_a_01077.

Tellegen, A., \& Atkinson, G. (1974). Openness to absorbing and selfaltering experiences ("absorption"), a trait related to hypnotic susceptibility. Journal of Abnormal Psychology, 83(3), 268.

Thibault, R. T., Lifshitz, M., \& Raz, A. (2017). Neurofeedback or neuroplacebo? Brain, 140(4), 862-864.

Turi, Z., Csifcsák, G., Boayue, N. M., Aslaksen, P., Antal, A., Paulus, W., \& Thielscher, A. (2019). Blinding is compromised for transcranial direct current stimulation at $1 \mathrm{~mA}$ for $20 \mathrm{~min}$ in young healthy adults. European Journal of Neuroscience, 50(8), 3261-3268.

van Elk, M. (2015). An EEG study on the effects of induced spiritual experiences on somatosensory processing and sensory suppression. Journal of the Cognitive Science of Religion, 2(2), 121-157.

Publisher's Note Springer Nature remains neutral with regard to jurisdictional claims in published maps and institutional affiliations. 